\title{
Media Streaming With Network Diversity
}

\section{Research indicates opportunities for improving media quality, in networks like the Internet with multiple paths, but also poses new coding, scheduling, routing, and path-computation problems.}

By Pascal Frossard, Senior Member IEeE, Juan Carlos de Martin, Member IEeE, and M. Reha Civanlar, Fellow IEEE

\begin{abstract}
Today's packet networks including the Internet offer an intrinsic diversity for media distribution in terms of available network paths and servers or information sources. Novel communication infrastructures such as ad hoc or wireless mesh networks use network diversity to extend their reach at low cost. Diversity can bring interesting benefits in supporting resource greedy applications such as media streaming services, by aggregation of bandwidth and computing resources. Typically, overlay network architectures compensate for lack of quality-of-service guarantees in the network by introducing redundancy in the media delivery system through network diversity. They can support efficient multimedia services when routing, coding, and scheduling algorithms are able to adapt to both the media information and the dynamic network status. This paper presents an overview of the distributed streaming solutions that profit from network diversity in order to improve the quality of multimedia applications. We discuss the coding techniques used for adaptive and flexible media streaming with network diversity. We describe the problem of media streaming with path diversity and focus on routing, path computation, and packet scheduling problems in multipath networks. Then, the advantages of server or source peer diversity in collaborative streaming solutions are discussed. Lastly, we present an overview of wireless mesh networks and focus on the typical constraints imposed by these novel communication models on media streaming with network diversity.
\end{abstract}

Manuscript received November 13, 2006; revised May 9, 2007.

P. Frossard is with EPFL, 1015 Lausanne, Switzerland (e-mail: pascal.frossard@epfl.ch).

J. C. de Martin is with Politecnico di Torino, 10129 Turin, Italy

(e-mail: demartin@polito.it).

M. R. Civanlar is with DoCoMo Labs, Palo Alto, CA 94304-1201 USA (e-mail: m.civanlar@ieee.org).

Digital Object Identifier: 10.1109/JPROC.2007.909876
KEYWORDS | Collaborative media streaming; distributed streaming; media overlay networks; mesh networks; multipath routing; multipath scheduling

\section{INTRODUCTION}

The past decade has shown the development of novel communication infrastructures, such as ad hoc and mesh networks, and peer-to-peer systems, which present the advantage of low deployment cost. They offer the possibility to extend the reach of the communication network where classical content delivery architectures cannot be deployed due to geographical or applicationspecific constraints such as client mobility. These packet network architectures typically construct network overlays so that they can compensate the lack of quality of service (QoS) by network diversity due to redundant sources and multiple communication paths to the client, as illustrated in Fig. 1. Network diversity presents several advantages for resource-greedy and delay-constrained services built on multimedia streaming. It supports the development of new multimedia communication applications by aggregation of bandwidth, storage or computing resources. At the same time, network diversity leads to a novel paradigm in media streaming, where all components actively participate in concert to improving the quality of stream received by the multimedia client.

This paper describes the novel opportunities but also the new coding and communication problems that are posed by distributed media delivery architectures. In particular, it highlights the benefits offered by network diversity that can be exploited to improve the quality of service offered to the streaming media client. We first discuss how novel coding algorithms can be designed to provide efficient media representations and enhanced 


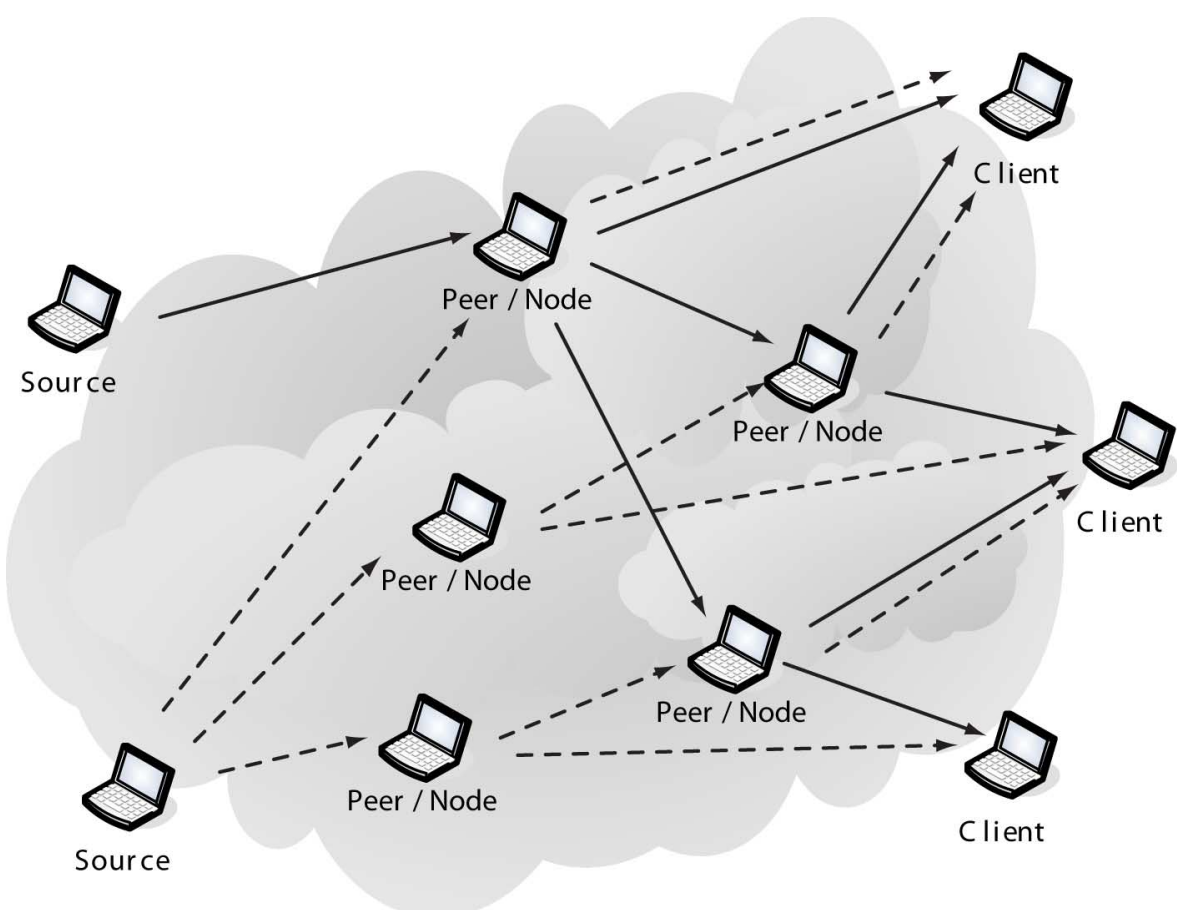

Fig. 1. Streaming architecture with network diversity. The media clients can connect simultaneously to several sources via multiple paths. The paths of data from the first and second sources are represented by solid and dashed arrows, respectively.

flexibility for distributed streaming algorithms. Then we illustrate how appropriate routing and distributed path computation, along with efficient packet scheduling, can be implemented to improve the media quality. Mediaspecific criteria have to be used in the path selection and rate allocation strategies in order to guarantee efficient solutions that are able to adapt to both the media content properties and the dynamic streaming architecture. In addition to path diversity, source or server diversity can also be judiciously exploited to yet improve the streaming experience. We also present collaborative streaming strategies that are able to coordinate packet scheduling in order to maximize the media quality.

Finally, we discuss distributed media streaming issues in the context of wireless mesh networks that represent one of the most popular solutions among the emerging communication systems with network diversity. They open a number of additional issues compared to traditional networks, such as the potentially high number of traversed hops, which may negatively affect the performance of real-time multimedia applications. At the same time, peculiar characteristics of mesh networks such as the presence of a potentially large number of densely interconnected nodes might be exploited by distributed streaming solutions to overcome the limitations caused by the unreliability of wireless channels and the highly dynamic behavior of network nodes. Recent research efforts have addressed the numerous challenges posed by wireless mesh networks, like routing, (auto)configuration, and self-healing strategies. However, limitation of the bandwidth, scarcity of wireless channels, and the multihop nature of connections still pose severe challenges for ensuring high-quality applications built on interactive multimedia communications.

The rest of this paper is organized as follows. Section II presents typical streaming problems in distributed architectures with network diversity and shows how network nodes can be arranged for efficient media delivery. Coding solutions adapted to redundant networks infrastructures, such as multiple description coding or channel coding, are presented in Section III. The problem of streaming with path diversity is addressed in detail in Section IV, where solutions for routing, distributed path computation, and packet scheduling are proposed. Section V presents collaborative streaming solutions, where distributed servers contribute together to improved media quality. Section VI describes emerging media delivery architectures and focuses on wireless mesh networks, with their specific characteristics in terms of capacity, latency, routing, and stability. Concluding remarks are finally given in Section VI.

\section{DISTRIBUTED STREAMING USING NETWORK DIVERSITY}

Distributed delivery architectures represent a scalable and cost-effective alternative to classic media delivery services, which permits to extend the reach of the network in the 
absence of IP multicast or expensive content distribution networks (CDNs). Their attractiveness mostly resides in their flexibility and self-organization, their inherent bandwidth scalability, and the redundancy in paths and source peers that provide robustness to network failures. Some fundamental differences between centralized infrastructures and distributed architectures such as mesh, ad hoc, or peer-to-peer systems, however, need to be addressed in order to offer efficient streaming solutions to media applications.

On the one hand, typical client-server architectures and $C D N s$ provide the network infrastructure that permits the deployment of generic media applications. In particular, they facilitate the implementation of tools for effective rich media delivery, like error correction, path computation, route choice, and rate adaptation. Such tools are generally built on the centralized computation paradigm that rely on important computational capabilities of streaming servers or proxy servers. On the other hand, distributed systems are in general less reliable but present the advantage of cheap service deployment (especially due to much lower bandwidth costs) and potential resource aggregation through multipath transmission. Distributed architectures lead to a streaming scenario where a single receiver connect to multiple network paths. The media packets are sent from different servers over (partially) disjoint separate network paths to a single client running at the receiver, as illustrated in Fig. 1. The client reconstructs the media stream with the packets that are received correctly from multiple sources and improves the quality of service thanks to the diversity of network resources. Such a scenario is a typical instance of the problem of communication over the multiple access channel. In terms of media data representation, it becomes a distributed coding problem where the availability of several sources results in improved media quality at the client. In addition, streaming with network diversity raises interesting issues in terms of routing and packet scheduling in order to ensure the timely delivery of the media information.

Without any guaranteed support from the distributed delivery architecture, streaming services rely on selforganized and adaptive network solutions in order to meet their stringent quality requirements. Intermediate nodes or peers that participate to the delivery of the media information from the sources to the clients are generally arranged following two main architectures: tree-based overlays for streaming sessions that disseminate the media content from media sources to a pool of client peers; and mesh overlay for massive parallel content distribution among peers. The first architecture organizes the network nodes as a single or multiple tree overlay that connects the source of the media content to the clients (Fig. 2). Clients are leaf nodes in the distribution tree, while intermediate peers push the content from the source towards the receivers. A peer can be a leaf in one or more distribution

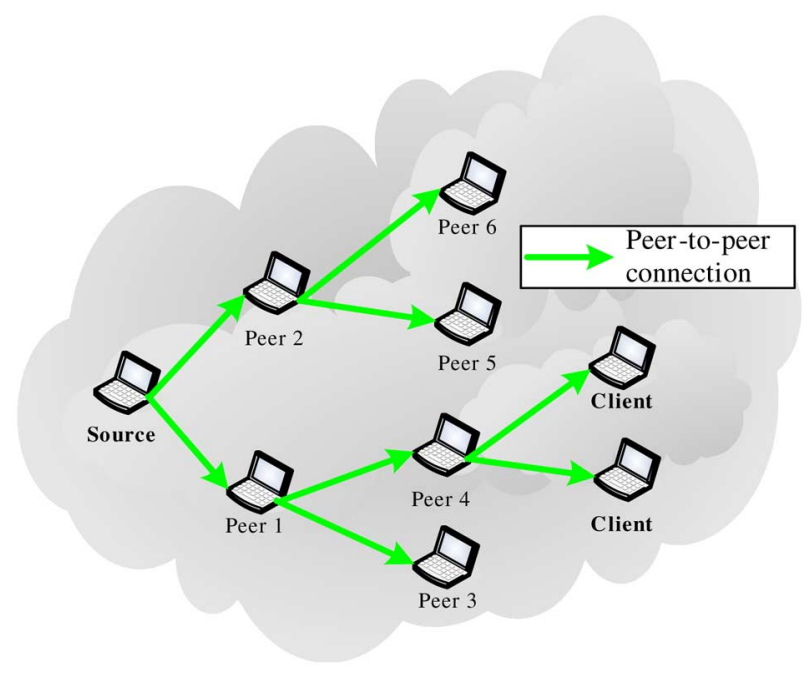

Fig. 2. Example of a tree architecture for media delivery with forwarding peers. The media information is generated by the server (root of the tree) and is transmitted along branches up to the media clients (leaves).

tree and at the same time an intermediate node in other trees. Single-tree architectures are easy to implement and to maintain, either in distributed or source-driven scenarios. In order to increase the stability and robustness to node failures, multiple-tree architectures can be implemented to provide redundancy in the network paths [1].

The mesh overlay architecture is based on the selforganization of nodes in a directed mesh (Fig. 3). The original media content from a source is distributed among different peers. A peer is connected to the mesh through one or more parent peers, from which it requests the media information, and to a set of child peers, where it forwards its media content. The inherent advantages of such an architecture reside mainly in the low cost and simplicity of structural maintenance and on the topology resilience due to the high number network paths. The above architectures are very common in peer-to-peer streaming systems or in solutions that use peer-to-peer overlays for media delivery [2], [3].

In both architectures, even with proper arrangement of peers, the streaming of media information stays quite complex due to the strict timing constraints and high bandwidth requirements. Multimedia data streams have very specific characteristics in terms of dynamic statistical properties and shall be sent on hardly predictable network channels. The delivery of video packets becomes, therefore, highly dependent on the actual state of the network and on the video characteristics. The design of effective media-streaming solutions over distributed architectures requires adaptive and robust strategies in order to fight against the variability and unreliability of the underlying transport medium. In the next sections, we provide an overview of the algorithms that permit one to adapt the 


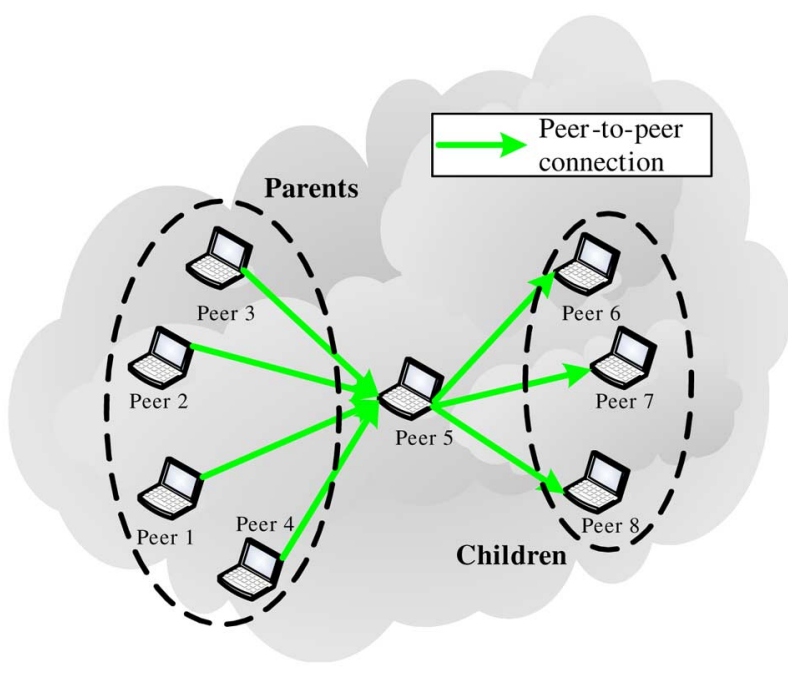

Fig. 3. Part of a mesh architecture for media delivery. Peers are interconnected and provide an overlay network for streaming with path diversity.

multimedia coding to the constraints and specificities of multipath networks, as well as effective routing and scheduling mechanisms.

\section{CODING FOR NETWORK DIVERSITY}

\section{A. Multiple Description Coding}

Many of the adaptive streaming techniques in the literature [4] can be elegantly combined with the diversity provided by distributed delivery architectures in terms of sources, paths, and channels. For example, scalable coding similar to the solution proposed in the recent MPEG SVC standard [5] are particularly adapted to streaming with network diversity. It encodes the multimedia information into hierarchical layers of different importance and permits a flexible transmission of video data on multiple network paths with different properties [6] along with a graceful quality degradation when resources become scarce.

Alternatively, multiple description coding (MDC) [7] offers a typical solution for media streaming with channel diversity offered by mesh or overlay networks. Since it permits one to avoid hierarchy between data layers, MDC naturally stands as a very interesting choice for adaptive and collaborative streaming on lossy communication channels. It is based on leaving a controlled degree of redundancy in the media descriptions so that decoders obtain a quality of service that is directly determined by the number of received packets. The best signal reconstruction is obtained when all descriptions are correctly received, while the correct reception of a single description already provides a reasonable quality [8].

Since the initial works on MDC for reliable communication over the telephone network [9], many interesting results have been reported in the information theory community that determine the multiple description ratedistortion region, which is the set of simultaneously achievable rates and distortions in MDC [10]-[12]. In image communication applications, MDC operates in the temporal, spatial, or frequency domain. In [13], the author proposes the multiple state video coding scheme, where the input video is split into sequences of odd and even frames. Each new sequence constitutes a description that is independently coded with its own prediction process (see Fig. 4). One description is sufficient to decode the stream at a reduced frame rate, and temporal error concealment can efficiently mask transmission errors. However, this scheme is penalized by a reduced coding efficiency due to the high redundancy between images in both coding threads. This is even exacerbated when the number of descriptions increases since the correlation between successive frames in the same description decreases. Alternatively, multiple description video coding can be based on spatial splitting [14] or on the multiple description scalar quantization framework proposed in [15]. Another important set of solutions resides in the application of unequal error protection coding for the generation of equivalent descriptions or media packets [16]. In fact, any layered coding can be converted into MDC by bundling the base layer with different enhancement layers while paying attention to the inter-layer dependencies. In general, ensuring synchronicity between the encoder and decoder states in case of loss is not a trivial issue in multiple description video coding, due to motion estimation. Some effective

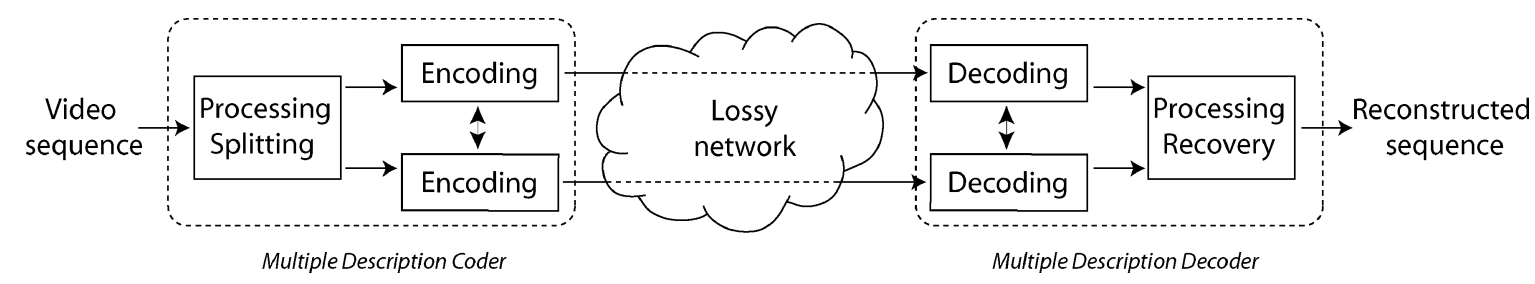

Fig. 4. Multiple state video coding system, where two encoding threads form two descriptions of the video sequence, which are later combined at the decoder. 
solutions based on distributed coding principles have however been proposed recently for MDC with reduced error propagation in the decoded video sequence [17]. A comprehensive overview of multiple description coding of video information is provided in [18], which also describes the benefits of MDC in multipath networks.

\section{B. Distributed Coding}

In scenarios with distributed collaborative servers, the key for efficient media communication strategies resides in the effective control of the redundancy between the different sources. Hence, the inherent problem in the use of multiple sources to send the same stream to a media client becomes the coordination between servers. In order not to waste resources with redundant data packets, servers have to carefully coordinate their packet scheduling strategies [19], generally with the help of the receiver. As a result, such distributed streaming systems may become overly complex and cumbersome, especially if conditions change on one of the source-client paths.

Distributed coding can be used as an alternative to complex scheduling algorithms in scenarios with server diversity. Network diversity actually provides an ideal framework for the application of distributed coding principles. They can be applied when several transmission paths are available between sender and receiver [20] and when several servers collaborate to an efficient quality of service at the media client. In particular, methods based on channel coding have been proposed to encode subparts (i.e., Group of Pictures (GOPs) and/or layers) of the video bitstream in order to prevent the need for precise coordination between servers [21], [22]. It mostly consists of smoothing out the difference in importance between media packets, so that the complexity due to packet scheduling can be avoided. At the same time, the encoder should still ensure that each transmitted packet is not redundant for the client that aggregates packets from the different servers. This is in spirit similar to [23]. A proper design of the channel code moreover permits to adapt to any kind of channel loss without requiring media transcoding at each sender [24].

For example, the substreams of a scalable video bitstream can be encoded using fountain or raptor codes [25] (Fig. 5). These codes belong to the family of rateless codes, which generates a high number of coded symbols from a set of $k$ source symbols. Any subset of $k+\epsilon$ raptor symbols (where $\epsilon$ is usually small) can then be used to decode the original $k$ source symbols with high probability. Therefore, the receiving client merely needs to retrieve $k+\epsilon$ symbols on aggregate from all available serving peers in order to decode the corresponding video segment. In particular, it is proposed in [21] to create one fountain per layer and per GOP of the original bitstream, as depicted in Fig. 5. The servers encode a set of source symbols whose size depends on the encoding rate of each layer and eventually send different packets to the client. The

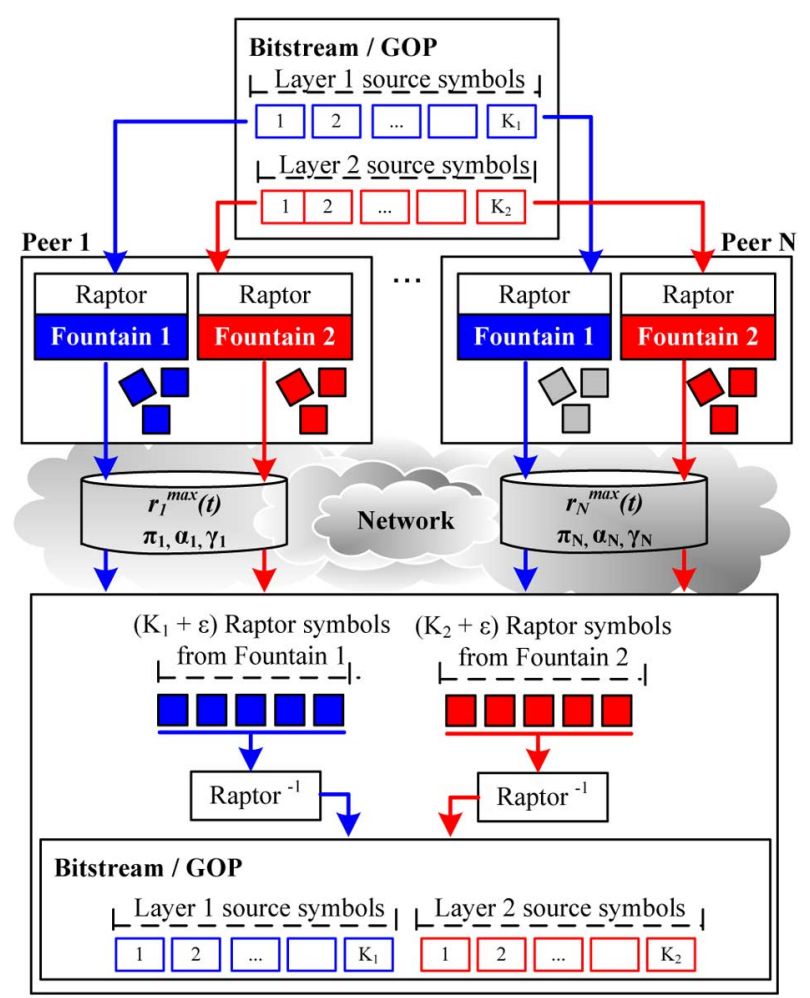

Client

Fig. 5. Distributed media coding with channel (fountain) codes. Each source peer encodes video layers in with different fountains, and the receiver only has to gather enough packets from the different sources to be able to decode the media information [21].

scheduling problem from the servers becomes trivial, since all packets in the same fountain have the same importance. The rate allocation problem consists in determining the optimal number of symbols to be sent from each server, such that the overall number of packets received at the client is maximized [21], [26]. Note that such a solution offers low decoding complexity and provides, along the way, a universal channel code for the transmitted stream. In addition, bitstreams encoded with raptor or channel codes, in general, present an increased resilience to loss. This certainly represents an important advantage in $\mathrm{p} 2 \mathrm{p}$ streaming systems with multiple source peers [27] that may however come at the price of an increased playback delay.

Finally, in addition to encoding algorithms such as multiple description or distributed coding implemented in the streaming servers, some coding operations can be implemented in the intermediate nodes and contribute to improving the performance of the streaming system [28]. In distributed architectures, these nodes can offer more than simple packet forwarding and rather filter or code media packets in order to increase the performance of the streaming system. In particular, network coding [29] have been proposed recently as an elegant solution for 
improving the reliability of multicast-based packet delivery. They increase the performance of the distributed streaming system by collaboration between intermediate nodes [27]. Such strategies lead to a novel communication paradigm represented by joint source and network coding problems, where sources and network peers together contribute to effective delivery of multimedia streams [30].

\section{MULTIPATH STREAMING}

\section{A. The Benefits of Path Diversity}

Path diversity in distributed communication infrastructures has fostered the development of effective streaming mechanisms to satisfy the requirements of media applications by aggregation of network resources. The early work presented in [31] and [32] establishes the generic framework for multipath streaming, which emerged as an effective solution to overcome some of the limitations of best effort packet networks. The specific advantages brought by the utilization of multiple transmission paths for media dissemination consist of aggregated network bandwidth, packet loss decorrelation, and delay reduction. The use of multiple paths permits one to increase the streaming bandwidth by balancing the load over multiple network paths between the media server and the client.

In the general framework offered by overlay networks such as peer-to-peer, mesh [33], or CDN architectures, the streaming client typically consumes the aggregated media from multiple transmission flows employed by the application. When properly combined with adaptive encoding solutions discussed above (e.g., forward error correction [34], multistream coding [35], or MDC [36], [37]), the definition of multiple end-to-end paths from the server to the client can dramatically improve the quality of service. Experimental work on multipath streaming [38] has offered some insight concerning the selection of content sources and streaming paths based on the jointness/disjointness of network segments.

A client in a wireless system can also aggregate the media information transmitted on multiple wireless channels [39]. Interference among transmission channels can be minimized by choosing non-overlapping wireless channels and by optimizing the transmission schedule in the wireless network [40]. Similarly, a mobile client in hybrid network scenarios can simultaneously benefit from multiple wireless services in order to retrieve the media information from a server that has a wired connection to the internet. In the rest of this section, we present in more details the problems that are typically associated with multi-path routing in order to take a maximum benefit from path diversity. These problems are media-specific path selection, distributed routing in large-scale networks, and packet scheduling. Most of the works in multipath streaming (e.g., [31]-[35], [37], [38], and [41]-[43]) rely on scenarios with two streaming paths that are defined a priori. However, the performance can yet be improved by considering a more generic framework where the choice of the optimal number of routes is performed jointly with proper rate allocation and packet scheduling.

\section{B. Path Selection for Media Streaming}

The selection of paths in distributed delivery infrastructures and the related rate allocation problems shall target an improved streaming experience measured in terms of video distortion. The system first needs to determine the available paths between server and receiver [44]. In ad hoc networks, for example, the source routing protocol (DSR) can be adapted such that it provides multiple viable paths for multimedia transmission [45]. When multiple paths are identified, the transmission policy becomes dependent on the rate allocation algorithm implemented in the streaming system. Several strategies have been proposed for efficient use of network resources, but many of them do not consider media specific metrics and rather single network parameters, like the overall throughput. Numerous routing algorithms have been proposed to optimize pure network QoS metrics [46] when paths are aggregated, or to improve the performance of transmission control protocol (TCP) over wireless ad hoc networks [47]. The optimization of network resource allocation in overlay multicasts has further been considered in [48], as well as in [49], where the authors concentrate on achieving fairness and maximize the network resource utilization for multicast information flows.

More generally, optimized streaming strategies however result from the joint optimization of multiple metrics (e.g., throughput, packet loss ratio, fairness, and delays). But, routing under multiple constraints is an NP-hard problem in general. Heuristic algorithms have been proposed for both source routing and hop-by-hop routing in order to find one path satisfying the QoS requirements of multimedia applications [50]. Recent works in routing with multiple constraints optimize a linear [51] and, respectively, a nonlinear [52] relation between network constraints using low-complexity algorithms. A similar function built on multiple path metrics is used in [53] to find multiple network paths for streaming. However, none of these works specifically considers the multimedia application characteristics in the routing decisions that are only based on network metrics. Unfortunately, the best paths found by classic routing algorithms are suboptimal from a media perspective in $30 \%-80 \%$ of the cases [54].

Clearly, media specific metrics have to be considered in the selection of paths, since maximum throughput does not always lead to optimal performance for media streaming applications that are typically sensitive to transmission delays. The early work in [38] derives a few empirical rules for path selection. These rules consider network metrics (e.g., available bandwidth, loss rate, and hop distance) and other media aware metrics (e.g., link jointness/disjointness, 


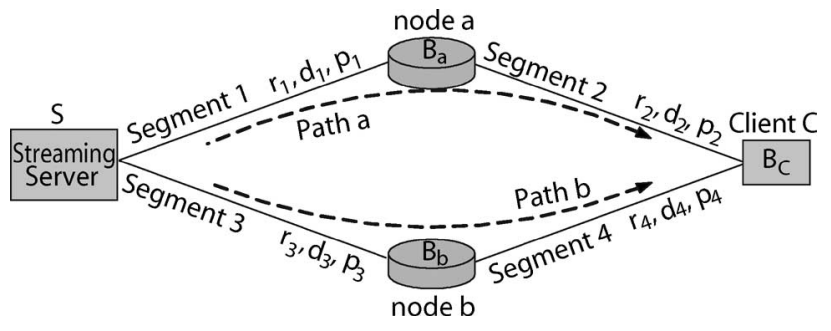

Fig. 6. Typical multipath streaming scenario. The client accesses the streaming server simultaneously through two different paths, each one composed of two segments (characterized by rate $r_{i}$, delayd $d_{i}$, and loss probability $p_{i}$ ) with intermediate buffers $\left(B_{i}\right)$. optimized performance is obtained by properly balancing the streaming rate on both paths.

video distortion). In general, one could use a generic end-toend video distortion metric, which encompasses both the source distortion and the channel distortion. The source distortion is mostly driven by the encoding or streaming rate and generally decays with increasing encoding rate. This decay is quite steep at low bit rate and becomes very slow at high bit rate. It also depends on the media sequence content, and the encoding quality is typically lower at a given encoding rate when the sequence is more complex to encode. The channel distortion is dependent on the average loss probability and the sequence characteristics. It is roughly proportional to the number of video entities (e.g., frames) that cannot be decoded. The end-to-end distortion can thus be written as $D=f(R, \pi, \Gamma)$, as it depends on the streaming rate $R$, the loss probability $\pi$, and sequencedependent parameters $\Gamma$. At low to medium bit rate, a commonly accepted model for the source rate distortion is a decaying exponential function of the encoding rate, while the channel distortion is proportional to the number of lost packets (or, equivalently, the packet loss probability when the number of packets per frame is independent of the bit rate) [55]. This model provides a simple approximation that follows quite closely the behavior of more sophisticated distortion measures, such as those proposed in [56] and [57]. Obviously, the optimization of the end-to-end distortion depends on network metrics like available bandwidth and loss probability. At the same time, media-specific parameters, such as the nature of the media information, or the type of encoding also have to be considered for proper rate allocation in multipath media streaming.

In more detail, it is clear that the total streaming rate $R$ and the end-to-end loss probability $\pi$ directly depend on the path selection and the flow rate allocation. In the multipath scenario described in Fig. 6, the media server can choose any rate allocation $\vec{\rho}=\left[\rho_{1}, \rho_{2}\right]$ that respects the maximum bandwidth constraints given by $\left(r_{1}, r_{2}\right)$. The total media streaming rate $R$ becomes simply the sum of the rates on each path, and the overall loss probability $\pi$ experienced by the media application can be computed as the average of the loss probabilities of the paths used for streaming. The optimal path selection and rate allocation consists in finding the best vector $\overrightarrow{\rho^{\star}}=\left[\rho_{1}^{\star}, \rho_{2}^{\star}\right]$, which minimizes the end-to-end distortion. In general, paths may, however, not be completely disjoint, and a rate-allocation vector $\vec{\rho}$ is a valid rate allocation on the network graph if and only if all flow rates can be simultaneously aggregated on all paths. While such an optimization problem is generally combinatorial, an algorithm whose complexity is linear with the number of available end-to-end paths can solve the optimal rate allocation problem in specific yet practical network topologies (i.e., topologies that can be split into independent subgraphs between server and client) [58]. When the characteristics of all paths are known by the server, the optimal rate allocation can be achieved in this case by a greedy path selection algorithm that uses first the paths affected by the smallest overall loss process. At the same time, the rate of bottleneck links that are shared by multiple network paths should also be split in a greedy manner among media flows.

Interestingly, the optimal resource allocation is not always based on network flooding when the media encoding strategy cannot be finely adapted to the loss process [58]; the appropriate selection of the total streaming rate depends on both the network and media stream characteristics. In order to illustrate the importance of rate allocation in multipath streaming, Fig. 7 presents the distribution of the relative end-to-end quality improvement for the optimal rate allocation when compared to heuristicbased algorithms, namely, i) a single path transmission scenario that selects the best path in terms of loss

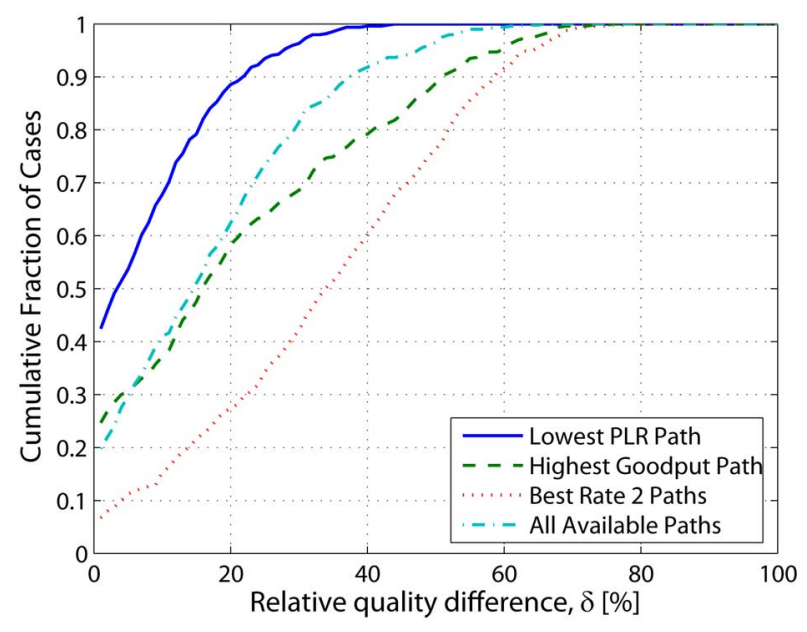

Fig. 7. Quality improvement of optimal versus heuristic rate allocation algorithms in random wireless mesh network topologies. From [58]. The results have been averaged over 500 random graphs, where any two nodes are directly connected with a probability of 0.6 , with a average total streaming rate of $R=450 \mathrm{kbps}$. The network topologies are representative of typical wireless mesh networks, and the parameters for each edge are randomly chosen according to a normal distribution, in the interval $\left[10^{5}, 710^{5}\right]$ bps for the bandwidth, and respectively $\left[10^{-3}, 41^{-2}\right]$ for the loss probability. 
probability, ii) a single path transmission scenario, which uses the best path in terms of effective bandwidth or "goodput," iii) a multipath transmission scenario that picks the best two paths in terms of goodput, and iv) a multipath transmission scenario that uses the maximum available number of flows. Since optimal rate allocation targets the maximization of a media distortion metric, it can be seen that it outperforms heuristic-based routing strategies that consider only network metric for routing and rate allocation. Finally, Fig. 7 also shows that flooding is not necessarily a good strategy when one has to transmit a video stream whose loss protection cannot be finely tuned to the error probabilities on the multipath network. The use of paths of very poor quality is not beneficial, except in the unlikely cases where such paths are used exclusively for pure redundancy packets.

One of the major drawbacks of most receiver- or senderdriven routing algorithms, however, lies in the need of full topology knowledge at a single point in the network, namely, the client or the server. This is required for an optimal decision in terms of source peer selection and path rate allocation. In large-scale networks, end-to-end traffic monitoring at a single peer becomes, however, cumbersome or inefficient. A solution for distributing the routing decisions among network nodes is proposed in [59]. Every intermediate peer takes an individual routing decision for every incoming packet, based only on local topology information. At the same time, all the peers forward information about the network status to the media client for coordination. Depending on the local path selection and rate allocation rule implemented at each intermediate node, the media application trades off the optimality of the average end-to-end quality, with flexibility and convergence time of the rate allocation in case of network fluctuations. In the case of distributed rate allocation, the routing also needs to consider a media-specific metric for an efficient solution in terms of end-to-end distortion. Media-specific rate allocation typically outperforms distributed heuristic routing strategy that simply forwards the packet on the best outgoing link.

\section{Scheduling in Multipath Streaming}

Once streaming paths and average rate allocation are defined, the streaming system still has to decide on the proper scheduling of the media packets. Packets of a media stream indeed do not all contribute evenly to the video quality at the receiving peer. Moreover, a packet is useful to the receiving peer only if $i$ ) it arrives before its decoding deadline and ii) all previous packets necessary to a correct decoding have been correctly received. The unequal importance of video packets, along with timing constraints, requires the derivation of efficient packet scheduling algorithms that determine which packets should be transmitted at a given time instant on a given streaming path, in order to maximize the overall streaming quality. Packet scheduling solutions have been widely studied in client- server architectures with a single channel. Rate-distortion optimized packet scheduling strategies [60]-[62] or frame discard strategies [63], [64] have been proposed to adapt the packet transmission to available bandwidth.

A few recent works, have considered specifically the problem of scheduling over multiple network paths. The multipath earliest deadline packet first (EDPF) algorithm has been proposed in [65] in order to solve the packet scheduling problem by computing the earliest delivery time for each packet, on each of the paths. By sending each packet on the path that ensures the earliest delivery at the client, the authors minimize the packet reordering cost. A selective frame discard strategy that drops less important frames has been proposed to adapt to the channel bandwidth [66]. More generic video formats (e.g., scalable coding), with improved granularity in media packets but also more complex packet dependencies, have been considered in [67]. A search algorithm is proposed for the optimal server-driven transmission policies for sets of sequential video packets, given the network scenario and client requirements. It does not only take advantage of the increased aggregated bandwidth of multiple network paths but it also benefits from the different paths to reduce the playback delay experienced by the client. A strategy based on load-balancing techniques leads to small quality variations on dynamic bandwidth channels and preserves a minimal quality level by improved scheduling. Interestingly enough, the performance of such a scheduling algorithm stays quite consistent for small video prefetch windows and for low accuracy in the channel bandwidth prediction. This becomes particularly interesting in multipath live streaming systems with stringent delay constraints and simple bandwidth prediction methods. Finally, it has to be noted that buffer constraints in the network nodes may actually have a nonnegligible impact on the scheduling strategy in multipath scenarios [67]. In the general case of network topologies with heterogeneous channel parameters, efficient packet scheduling strategies thus have to take into account the capabilities of the forwarding peers.

\section{STREAMING WITH SERVER DIVERSITY}

With the development of distributed network infrastructures, collaborative streaming becomes an interesting solution to increase the performance of multimedia applications. The distortion perceived at the media clients can be minimized with the availability of several sources that collaborate for increased resources or improved reliability (see Fig. 8). The client can simultaneously access the same multimedia data information at multiple peers in the network, similar to the digital fountain model [68], where the system tries to minimize the download time of a file at a client by connecting to multiple mirror server sites. The possibility of receiving the same data over multiple paths increases the resilience of the media presentation to network outages or congestion onsets. At the same time, it 


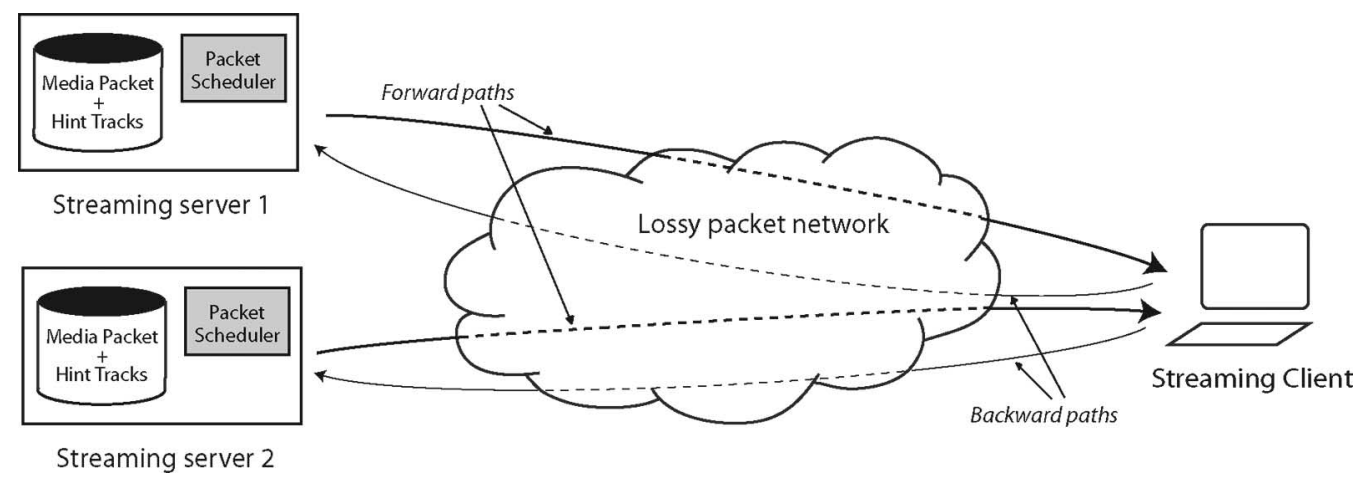

Fig. 8. Framework for distributed streaming with packet erasure channels.

reduces the startup delay of the client application and provides smooth and continuous media playout at the decoder.

The source peer selection and the rate allocation problems are typically addressed in receiver-driven streaming scenarios, where the client coordinates the streaming process. Content location information can be accessed by the receiver at supernodes/servers or from other peers (e.g., by search algorithms adapted to fully decentralized systems). Furthermore, the receiver can probe for network connection information towards candidate source nodes. Based on network information and streaming session characteristics, the receiver makes an informed choice of source nodes and network transmission paths [37]. Also, it is possible to design a congestion-preventing solution by adjusting the transmission rate of each participating source based on the TCP-friendly rate available [36]. Application adaptation can then be commanded by the receiver in order to reflect the changes observed in the transport medium (e.g., via RTP/RTCP statistics). Unsurprisingly, the choice of the lowest error paths first is generally advocated for the media delivery from multiple source nodes to the receiving end [43], similarly to the discussion proposed in the previous section.

Efficient delivery from multiple sources relies on a proper control of packet redundancies so that the client does not receive multiple copies of the same data. This would result in a waste of resources. The earliest work that has studied the problem of transmission coordination among multiple senders in distributed streaming is [69]. The authors propose an algorithm that runs at the client and performs rate allocation and packet partitioning among the senders. This algorithm can be further combined with forward error correction for improved error resilience to packet loss [70]. For optimal performance, the receiver-driven control protocols shall synchronize the senders' transmissions in a rate-distortion optimized way by selecting in priority the packets the bring the largest improvement in quality per increment of streaming rate [71]. For improved error resilience, MDC, discussed in the previous sections, can be employed at each sender to encode scalable media content that is streamed afterwards to the client [36], [72], [73]. The number of descriptions as well as their rates and redundancy levels among descriptions can also be adjusted in real time so that each peer can adapt its transmission rate to bandwidth constraints.

While most of the works in distributed streaming consider that the receiver selects packets and streaming servers, implementing a completely distributed video packet scheduling algorithm remains a complex task. Ideally, distributed algorithms run independently on each source peer but unanimously decide the set of video packets to be sent along with the disjoint partitions allocated to each transmitting peer. Sender-driven schemes provide several advantages over receiver-driven solutions. Optimization of the media quality is facilitated, since the relative importance of the media packets is known at the servers. In addition, the deployment of practical solutions is facilitated since the management of overall network resources becomes easier. An optimization framework for sender-driven streaming is proposed in [74] and [75], where multiple servers synchronize their transmission schedules for sending a standard (single description) video stream without information exchange besides bandwidth information estimated at the client. The bandwidth estimates are used in conjunction with a packet scheduling optimization framework to compute appropriate transmission actions at each sender. In order to reduce the computational load imposed by the optimization framework on each sender, an alternative technique can be designed with a priori packet classification according to their relative importance [76], [77]. The complexity of taking joint decisions among servers is thus alleviated by partitioning beforehand the set of available packets among the potential serving peers, which provides interesting solutions for practical and scalable implementations of adaptive and efficient distributed streaming systems [74]. The average rate-distortion performance of distributed streaming with two servers is represented in Fig. 9. It shows that the consideration of media packet importance offers the ability 


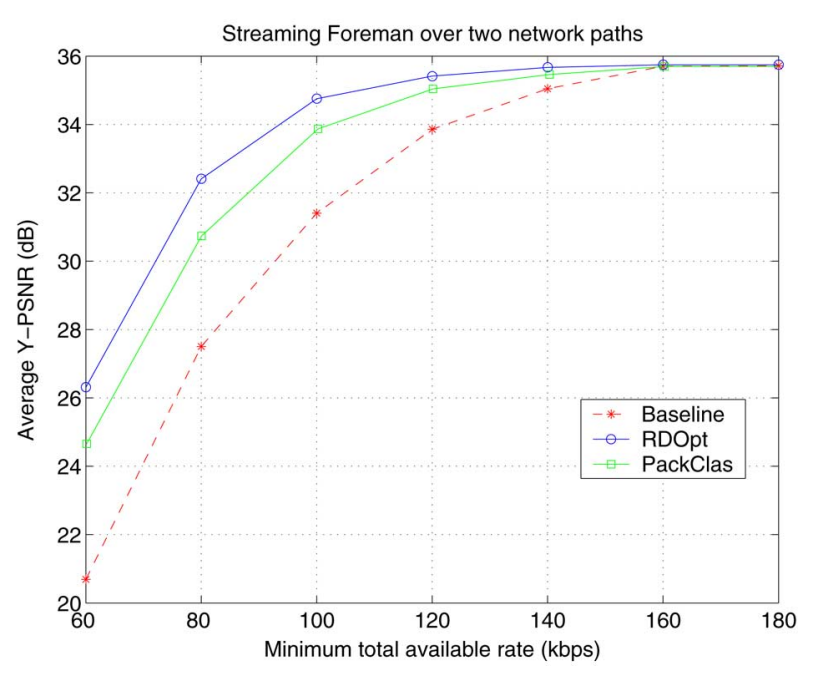

Fig. 9. A verage rate-distortion performance for distributed streaming of Foreman common intermediate format video sequence (H.264 encoding, with constant bit rate), for a rate-distortion optimal solution (RDOpt), a strategy based on a packet partitioning (PackClas), and a baseline solution with allocation proportional to available bandwidth (Baseline). From [74].

to outperform a conventional system that implements proportional packet scheduling based only on the relative available bandwidth values. Finally, source coordination can further be facilitated by distributed coding strategies that smooth the difference in importance that exist in media packet streams, as discussed in Section III-B.

\section{EMERGING WIRELESS DELIVERY ARCHITECTURES}

\section{A. Wireless Mesh Networks}

In this section, we present a typical framework for distributed streaming with network diversity, where the algorithms presented above find a straightforward application. Structured media delivery architectures such as content distribution networks still represent the typical infrastructure for streaming service with controllable quality. They are, however, quite expensive to set up, and their deployment is often contingent to good network accessibility. These are among the reasons that explain the recent proposals of novel delivery architectures that extend the reach of the network and permit easy and cheap deployment of new streaming applications. These new infrastructures try to augment the resources offered to the application by aggregation of bandwidth, storage, or cheap computing resources. At the same time, they pose several new problems for the development of effective media-streaming solutions that often have to cope with the high variability of such systems.

Among these novel infrastructures, wireless mesh networks (WMNs) have emerged as a key technology for a variety of new applications that require flexible network support. As an evolution of multihop mobile ad hoc wireless networks (MANETs), the so-called mesh network configuration maintains the ad hoc communication structure but consists of two architectural levels: mesh routers and mesh clients. Mesh routers have minimal mobility and form the WMN backbone (see Fig. 10). Meshed networks can serve as indoor or outdoor networks. For example, municipalities might wish to create their own wireless network infrastructure, or meshes may also serve as outdoor portions of campus networks. Multimedia communications can greatly benefit from this new kind of infrastructure, as WMN may offer greater bandwidth at lower cost when compared to third-generation cellular networks. Compared to the classical Internet infrastructure, connections in wireless networks are more unstable and channels are more dynamic and prone to network interference. While data flows such as file transfer may be almost arbitrarily curtailed and still be useful, multimedia communications are, however, quite demanding in terms of quality of service. Channel availability and network latency problems become quite important in $\mathrm{WMN}$, especially when the size and complexity of multiple hop mesh networks increase. If delay, bandwidth, or packet loss rate are too constraining, delivery of voice or video packets may even be of no use. The adaptive coding and streaming methods proposed in the previous section hence become crucial for effective multimedia communication applications in emerging wireless mesh networks.

In the rest of this section, we further discuss the specificities of wireless mesh networks for the implementation of distributed streaming solutions. We focus our discussion on 802.11 networks that appear to be the most promising networking technology for multimedia services over WMNs [78], [79]. The IEEE 802.11 Working Group is very active in the standardization of new interoperable 802.11-based standards that provide some interesting capabilities for multimedia communications such as bit rates on the order of $100 \mathrm{Mbps}$, QoS support, fast handoff,

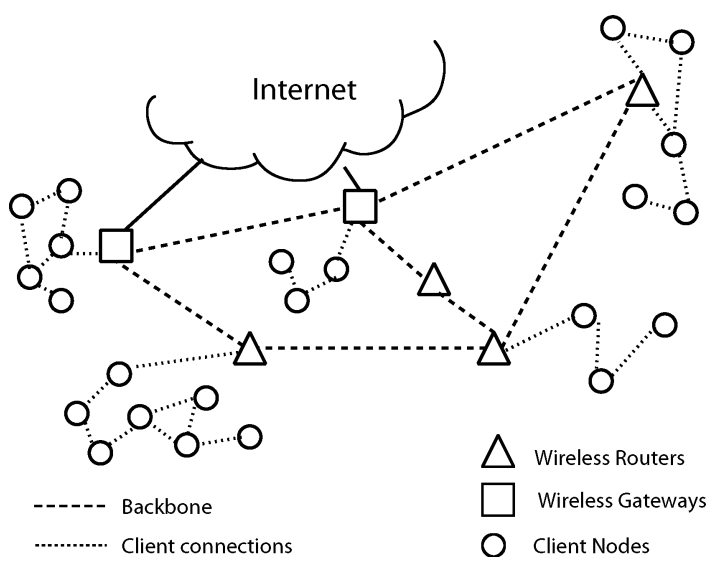

Fig. 10. An example of a wireless mesh network architecture. 
and mesh functionalities (e.g., the 802.11 standards). Other work has been initiated by the 802.16 standard committee for Medium Access Control (MAC) layer mesh extensions of the WiMax point-to-multipoint architecture [80]. Additional research and standardization work, however, is needed to bring the full benefits of mesh architecture to $802.16 /$ WiMax [81]. Solutions based on 802.11 still appear to be more easily deployable because they are widely available and operate in unlicensed cost-free frequency bands. Nevertheless, Wi-Fi and WiMax might be eventually integrated together; 802.16 wireless links can introduce additional capacity in the mesh, expand the network coverage [82], and hence offer increased flexibility for the deployment of multipath streaming solutions.

\section{B. Network Capacity and Latency}

Wireless networks based on the 802.11 standards are widely deployed in homes, enterprises, and public hot spots. Maximum nominal data rates vary from $11 \mathrm{Mbps}$ for 802.11b to $54 \mathrm{Mbps}$ for the 802.11g and 802.11a standards. An additional task group, $802.11 \mathrm{n}$, is working on higher maximum data rates, estimated in a theoretical value of 100-200 Mbit/s [83] using multiple transmitter and receiver antennas (multiple-input multiple-output technology). However, the maximum achievable throughput for 802.11 networks is far lower than the nominal data rate due to the nature of the wireless channel. For example, in $802.11 \mathrm{~b}$, the maximum experimental throughput is about 6.2 Mbit/s [84], a value that decreases as more stations are connected.

First-generation wireless network architectures were based on infrastructure access points or on direct communication between nodes. Nowadays, network nodes are gaining the ability to freely connect among themselves operating not only as a host but also as a router. That is, they can forward packets on behalf of other nodes that may not be within the direct wireless transmission range of their destination. As a consequence, in a mesh network, a packet destined to a node in the network may hop through multiple nodes to reach its destination. Analysis of the capacity of such networks [85] shows that they suffer from scalability issues, i.e., when the size of the network increases, their capacity degrades significantly with the increasing number of nodes.

Moreover, research has demonstrated that a node should communicate with nearby nodes only in order to maximize the network performance in terms of bandwidth [86]. But the large number of consecutive hops required to deliver the packets may severely limit the quality of service experienced by real-time multimedia applications, especially with regards to performance metrics such as end-toend delay, jitter, and packet loss ratio. Latency of several milliseconds per hop due to processing or transmission delay may preclude delay-intolerant applications such as voice and real-time interactive video after a few hops only. This problem is mainly due to the single-radio channel nature of early generation mesh networks where each node operates in half-duplex mode and shares the same radio frequency, i.e., all radios on the same channel must remain silent until the packet completes its hops within the same collision domain. Use of more sophisticated (and more expensive) multiple-radio mesh networks can increase the system scalability with the creation of a wireless "backbone," called backhaul network, which interconnects all nodes and handles traffic between nodes [87]. While excessive hop counts can sometimes be minimized by proper network architecture design, this is not the case for spontaneous, unstructured, ad hoc mesh networks that rather require support at the application level to mitigate the effect of excessive delay and jitter. It therefore outlines the importance of the number of hops that becomes a crucial criteria in the design of efficient routing and media rate allocation strategies.

\section{Network Routing}

Routing protocols are in charge of maintaining information on the topology of the network in order to calculate routes for packet forwarding. They influence the number of hops that user traffic must traverse to reach its destination along with other parameters such as the network topology, the length of the links or the wireless technology. Considerable research has addressed the problem of routing specific to wireless multihop networks [88]. The routing mechanism may choose to use information about the underlying topology of the network to collect the count of hops or distances of each node to all the other nodes or to determine where nodes are connected to each other. Some proposals utilize the shortest hop count metric as the path selection metric. This metric has been shown to result in poor network throughput because it favors long, low-bandwidth links over short, high-bandwidth links [89]. More recent proposals aim instead to improve routing performance by utilizing routeselection metrics [90], which consider not only the throughput but also the contribution of both bandwidth and delay. A combination of several metrics along with their participation to the end-to-end distortion of the media application, is however necessary for optimal routing and rate allocation strategies, as has been shown in Section IV.

Naturally, the mesh topology also enables the definition of multiple routes between two endpoints. Such routes may be utilized (together with the previously proposed solutions) by multipath routing techniques to increase the quality of service in multimedia transmission. If the current path becomes unusable, the traffic flow can then quickly switch to one of the alternate paths without waiting for setting up a new routing path. The existence of multiple paths can also help to reduce the chance of interrupting the service due to node mobility [92]. Even better, data partitioning over multiple paths can reduce the short-term correlation in real-time traffic and therefore improve the performance of multimedia streaming 
applications, since burst losses in general cause more important degradations in the video stream quality [91]. Clearly, monitoring of several available paths is necessary for ensuring a sustained quality of service [93].

\section{Handoff}

Finally, one of the typical problems of wireless mesh networks with mobile peers is handoff management. As shown in Fig. 10, one of the main characteristics of mesh networks is that they have only a few wireless gateways connected to a wired network while the wireless routers (WRs) provide network access to mobile clients (i.e., they act as access points to the clients). The client may move freely within the range of a given WR. But as it moves away from a WR and gets closer to another WR, it should hand off all its open connections to the new one in order to preserve network connectivity. Ideally, the handoff should be completely transparent to mobile clients with no interruption, loss of connectivity, or transmission "hiccups."

In cellular data and voice systems, the handoff problem is typically coordinated by the network itself using signaling embedded in the low-level protocols that are able to leverage considerable information about the network topology and client proximity. In contrast, 802.11 networks currently lack efficient and transparent handoff solutions. Consequently, as a mobile 802.11 client reaches the limits of its current coverage region inside the mesh, it must abandon its current WR, actively probe the network to discover alternatives, and then reconnect to the current best WR. Such delays may be penalizing for streaming applications with strict timing constraints. Similarly, as one cannot know in advance if necessary QoS resources are available at a new access point, a transition can lead to poor application performance. And forcing an additional session at the new access point may even result into degrading ongoing connections. As the need for admission and congestion control becomes apparent, the 802.11e standard [94] specifically deals with QoS for wireless networks by means of a component called Wi-Fi multimedia. The improvements continue in the developing standard 802.11n.

Multihop ad hoc networking, high traffic load, lack of coordination among nodes, and no facility for route reservation or clustering contribute together to build a challenging framework for real-time multimedia communications in WMNs. Future advances in the wireless protocols along with appropriate distributed coding and streaming solutions, will surely enable the deployment of resource greedy and delay critical multimedia applications in wireless environments.

\section{CONCLUSIONS}

The rapid development of novel delivery architectures has recently opened interesting research problems for the distributed delivery of multimedia streams. Novel delivery architectures such as wireless mesh networks permit one to easily extend the reach of the network and to provide increased streaming performance by resource aggregation. The diversity offered by such infrastructures however raises a number of interesting questions in the coding of the media information, the routing and rate allocation on multipath networks, or the collaborative streaming from distributed server peers. They permit one to improve the user experience by solutions that are adaptive to the media information and to dynamic network variations. Research and development efforts are, however, still necessary before actual media streaming services can be efficiently offered on wireless mesh networks. In particular, the development of highly interactive applications such as gaming and video conferencing or the deployment of multiple simultaneous sessions outline the importance of rate allocation solutions for a proper distribution of resources among all the peers in the systems. The efficient utilization of power resources in wireless systems opens another interesting research path. Finally, security still represents a crucial problem in highly distributed delivery systems and probably remains an important factor that slows down the deployment of rich media applications over uncontrolled network environments.

\section{Acknowledgment}

The authors would like to thank J. Chakareski, D. Jurca, E. Masala, and J.-P. Wagner for providing figures and helpful contributions to the text.

\section{REFERENCES}

[1] V. N. Padmanabhan, H. J. Wang, and P. A. Chou, "Resilient peer-to-peer streaming," in Proc. IEEE ICNP, Atlanta, GA, 2003.

[2] C. Yi, L. Baochun, and K. Nahrstedt, "oStream: Asynchronous streaming multicast in application-layer overlay networks," IEEE J. Sel. Areas Commun., vol. 22, no. 1, pp. 91-106, 2004.

[3] X. Zhang, J. Liu, B. Li, and T. S. P. Yum, "CoolStreaming: A data-driven overlay network for peer-to-peer live media streaming," in Proc. IEEE INFOCOM 2005, vol. 3, pp. 2102-2111.
[4] J. Chakareski and P. Frossard, "Adaptive systems for improved media streaming experience," IEEE Commun. Mag., Jan. 2007.

[5] H. Schwarz, D. Marpe, and T. Wiegand, "Overview of the scalable video coding standard," IEEE Trans. Circuits Syst. Video Technol., to be published.

[6] C. Jiancong, S. H. G. Chan, and V. O. K. Li, "Multipath routing for video delivery over bandwidth-limited networks," IEEE J. Sel. Areas Commun., vol. 22, no. 10, pp. 1920-1932, 2004.

[7] V. K. Goyal, "Multiple description coding: Compression meets the network," IEEE Signal Process. Mag., vol. 18, pp. 74-93, Sep. 2001.

[8] J. Apostolopoulos and M. Trott, "Path diversity for enhanced media streaming,"
IEEE Commun. Mag., vol. 42, pp. 80-87, Aug. 2004.

[9] S. E. Miller, "Fail-safe transmission without standby facilities," Bell Labs, Tech. Rep. TM80-136-2, Aug. 1980.

[10] A. A. El Gamal and T. M. Cover, "Achievable rates for multiple descriptions," IEEE Trans. Inf. Theory, vol. IT-28, pp. 851-857, Nov. 1982.

[11] L. Ozarow, "On a source-coding problem with two channels and three receivers," Bell Syst. Tech. J., 1980.

[12] Z. Zhang and T. Berger, "New results in binary multiple descriptions," IEEE Trans. Inf. Theory, vol. IT-33, pp. 502-521, Jul. 1987. 
[13] J. G. Apostolopulous and S. J. Wee, "Unbalanced multiple description video communication using path diversity," in Proc. IEEE ICIP, Oct. 2001, pp. 966-969.

[14] N. Franchi, M. Fumagalli, R. Lancini, and S. Tubaro, "Multiple description video coding for scalable and robust transmission over IP," IEEE Trans. Circuits Syst. Video Technol. vol. 15, no. 3, pp. 321-324, Mar. 2005.

[15] C. Tian and S. S. Hemami, "Sequential design of multiple description scalar quantizers," in Proc. IEEE DCC, Mar. 2004, pp. 32-41.

[16] R. Puri and K. Ramchandran, "Multiple description source coding using forward error correction codes," in Proc. 33rd Asilomar Conf. Signals, Syst., Comput., 1999, vol. 1, pp. 342-346, paper 96.

[17] A. Jagmohan, A. Sehgal, and N. Ahuja, "Two-channel predictive multiple description coding," in Proc. IEEE ICIP, 2005, vol. 2, pp. II-670-II-673.

[18] W. Yao, A. R. Reibman, and L. Shunan, "Multiple description coding for video delivery," Proc. IEEE, vol. 93, no. 1, pp. 57-70, 2005.

[19] V. Agarwal and R. Rejaie, "Adaptive multi-source streaming in heterogeneous peer-to-peer networks," in Proc. Multimedia Comput. Network. (MMCN 2005), Jan. 2005.

[20] V. Stankovic, Y. Yang, and Z. Xiong, "Video multicast over heterogeneous networks based on distributed source coding principles," in Proc. IEEE ICIP, 2006.

[21] J.-P. Wagner, J. Chakareski, and P. Frossard, "Streaming of scalable video from multiple servers using rateless codes," in Proc. IEEE ICME, Toronto, ON, Canada, 2006.

[22] T. Schierl, C. Hellge, K. Ganger, T. Stockhammer, and T. Wiegand, "Multi source streaming for robust video transmission in mobile ad-hoc networks," in IEEE Int. Conf. Image Process., Atlanta, GA, Oct. 2006, pp. 1669-1672.

[23] C. Wu and B. Li, "rStream: Resilient peer-to-peer streaming with rateless codes," in Proc. ACM Multimedia 2005, Nov. 2005.

[24] A. Majumdar, R. Puri, and K. Ramchandran, "Distributed multimedia transmission from multiple servers," in Proc. IEEE ICIP, 2002.

[25] A. Shokrollahi, "Raptor codes," IEEE Trans. Inf. Theory, vol. 52, no. 6, pp. 2551-2567, 2006.

[26] J.-P. Wagner and P. Frossard, "Adaptive and robust media streaming over multiple channels with bursty losses," in Proc. EUSIPCO, Poznan, Poland, 2007.

[27] C. Wu and B. Li, "rStream: Resilient peer-to-peer streaming with rateless codes," Proc. ACM Multimedia, pp. 307-310, 2005.

[28] S. Karande, M. Wu, and H. Radha, "Network embedded FEC (NEF) for video multicast in presence of packet loss correlation," in Proc. IEEE ICIP, 2005.

[29] N. Ahlswede, R. Cai, S. Li, and R. Yeung, "Network information flow," IEEE Trans. Inf. Theory, vol. 46, pp. 1204-1216, Jul. 2000.

[30] S. Yufeng, S. Kalyanaraman, J. W. Woods, and I. V. Bajic, "Joint source-network error control coding for scalable overlay video streaming," in Proc. IEEE ICIP, 2005, vol. 1, pp. I-177-I-180.

[31] L. Golubchik, J. Lui, T. Tung, A. Chow, and W. Lee, "Multi-path continuous media streaming: What are the benefits?" ACM $J$. Perform. Eval., vol. 49, no. 1-4, pp. 429-449, Sep. 2002.

[32] Y. Li, S. Mao, and S. S. Panwar, "The case for multipath multimedia transport over wireless ad hoc networks," Proc. IEEE/ACM BroadNets, pp. 486-495, Oct. 2004.

[33] A. C. Begen, Y. Altunbasak, M. R. Civanlar, and G. Gorbil, "High-resolution video streaming in mesh-networked homes," in Proc. IEEE ICIP, 2005, vol. 1, pp. I-181-I-184.

[34] T. Nguyen and A. Zakhor, "Path diversity with forward error correction (pdf) system for packet switched networks," in Proc. IEEE INFOCOM, 2003, vol. 3, pp. 663-672.

[35] S. Mao, S. Lin, S. S. Panwar, Y. Wang, and E. Celebi, "Video transport over ad hoc networks: Multistream coding with multipath transport," IEEE J. Sel. Areas Commun., vol. 21, pp. 1721-1737, Dec. 2003.

[36] E. Akyol, A. M. Tekalp, and M. R. Civanlar, "A flexible multiple description coding framework for adaptive peer-to-peer video streaming," IEEE J. Sel. Topics Signal Process., vol. 1, pp. 231-245, Aug. 2007.

[37] A. C. Begen, Y. Altunbasak, O. Ergun, and M. H. Ammar, "Multi-path selection for multiple description video streaming over overlay networks," Signal Process. Image Commun., vol. 20, pp. 39-60, 2005.

[38] J. Apostolopoulos, T. Wong, W. Tan, and S. Wee, "On multiple description streaming with content delivery networks," in Proc. IEEE INFOCOM, Jun. 23-27, 2002, vol. 3, pp. 1736-1745.

[39] R. Chandra, P. Bahl, and P. Bahl, "Multinet: Connecting to multiple ieee 802.11 networks using a single wireless card," in Proc. IEEE INFOCOM, 2005, vol. 2, pp. 882-893.

[40] P. von Rickenbach, S. Schmid, R. Wattenhofer, and A. Zollinger, "A robust interference model for wireless ad-hoc networks," in Proc. IEEE WMAN'05, 2005.

[41] J. Kim, R. M. Mersereau, and Y. Altunbasak, "Network-adaptive video streaming using multiple description coding and path diversity," in Proc. IEEE ICME, 2003.

[42] X. Zhu and B. Girod, "Distributed rate allocation for multi-stream video transmission over ad-hoc networks," in Proc. IEEE ICIP, 2005.

[43] T. Nguyen and A. Zakhor, "Multiple sender distributed video streaming," IEEE Trans. Multimedia, vol. 6, pp. 315-326, Apr. 2004.

[44] S. Vutukury and J. J. Garcia-Luna-Aceves, "Mdva: A distance-vector multipath routing protocol," in Proc. IEEE INFOCOM, 2001 vol. 1, pp. 557-564.

[45] W. Wei and A. Zakhor, "Robust multipath source routing protocol (RMPSR) for video communication over wireless ad-hoc networks," in Proc. IEEE ICME, 2004.

[46] J. L. Sobrinho, "Algebra and algorithms for qos path computation and hop-by-hop routing in the internet," IEEE/ACM Trans. Network., vol. 10, pp. 541-550, Aug. 2002.

[47] T. Murakami, M. Bandai, and I. Sasase, "Split multi-path routing protocol with load balancing policy (smr-lb) to improve TCP performance in mobile ad-hoc networks," IEICE Trans. Commun., vol. E89-B, no. 5, pp. 1517-1525, 2006.

[48] Y. Cui, Y. Xue, and K. Nahrstedt, "Optimal resource allocation in overlay multicast," IEEE Trans. Parallel Distrib. Syst., vol. 17, no. 8, pp. 808-823, Aug. 2006.

[49] S. Sarkar and L. Tassiulas, "A framework for routing and congestion control for multicast information flows," IEEE Trans. Inf. Theory, vol. 48, pp. 2690-2708, Oct. 2002.

[50] Z. Wang and J. Crowcroft, "Quality-of-service routing for supporting multimedia applications," IEEE J. Sel. Areas Commun., vol. 14, pp. 1228-1234, Sep. 1996.
[51] Y. Cui, K. Xu, and J. Wu, "Precomputation for multi-constrained QoS routing in high-speed networks," in Proc. IEEE INFOCOM, 2003, vol. 2, pp. 1414-1424.

[52] T. Korkmaz and M. M. Krunz, "Routing multimedia traffic with qos guarantees," IEEE Trans. Multimedia, vol. 5, pp. 429-443, Sep. 2003.

[53] Z. Ma, H.-R. Shao, and C. Shen, "A new multi-path selection scheme for video streaming on overlay networks," in Proc. IEEE ICC, 2004.

[54] S. Savage, A. Collins, and E. Hoffman, "The end-to-end effects of internet path selection," in Proc. ACM SIGCOMM, 1999, pp. 289-299.

[55] D. Jurca, S. Petrovic, and P. Frossard, "Media aware routing in large scale networks with overlay," in Proc. IEEE ICME, Jul. 2005.

[56] Y. J. Liang, J. G. Apostolopoulos, and B. Girod, "Analysis of packet loss for compressed video: Does burst-length matter?" in Proc. IEEE ICASSP, 2003.

[57] K. Stuhlmuller, N. Farber, M. Link, and B. Girod, "Analysis of video transmission over lossy channels," IEEE J. Sel. Areas Commun., vol. 18, pp. 1012-1032, Jun. 2000.

[58] D. Jurca and P. Frossard, "Media-specific rate allocation in multipath networks," IEEE Trans. Multimedia, vol. 9, no. 6, pp. 1227-1240, Oct. 2007.

[59] D. Jurca and P. Frossard, "Distributed media rate allocation for media in overlay networks," in Proc. IEEE ICME, 2006.

[60] P. A. Chou and Z. Miao, "Rate-distortion optimized streaming of packetized media," IEEE Trans. Multimedia, vol. 8, pp. 390-404, Apr. 2006.

[61] M. Roder, J. Cardinal, and R. Hamzaoui, "Branch and bound algorithms for rate-distortion optimized media streaming," IEEE Trans. Multimedia, vol. 8, pp. 170-178, Feb. 2006.

[62] D. Tian, X. Li, G. Al-Regib, Y. Altunbasak, and J. Jackson, "Optimal packet scheduling for wireless streaming with error-prone feedback," in Proc. IEEE WCNC, Mar. 21-25, 2004, vol. 2, pp. 1287-1292.

[63] Z.-L. Zhang, S. Nelakuditi, R. Aggrawal, and R. P. Tsang, "Efficient selective frame discard algorithms for stored video delivery across resource constrained networks," Real Time Imag., vol. 7, pp. 255-273, 2001.

[64] J. Huang, C. Krasic, and J. Walpole, "Adaptive live video streaming by priority drop," in Proc. Packet Video Workshop, 2003.

[65] K. Chebrolu and R. Rao, "Bandwidth aggregation for real-time applications in heterogeneous wireless networks," IEEE Trans. Mobile Comput., vol. 5, pp. 388-403, Apr. 2006.

[66] K. Chebrolu and R. R. Rao, "Selective frame discard for interactive video," in Proc. IEEE ICC, 2004, pp. 4097-4102.

[67] D. Jurca and P. Frossard, "Video packet selection and scheduling for multipath streaming," IEEE Trans. Multimedia, vol. 9, no. 3, pp. 629-641, Apr. 2007.

[68] J. Byers, M. Luby, and M. Mitzenmacher, "Accessing multiple mirror sites in paralel: Using tornado codes to speed up downloads," in Proc. IEEE INFOCOM, New York, NY, Mar. 1999, vol. 1, pp. 275-283.

[69] T. Nguyen and A. Zakhor, "Distributed video streaming over internet," in Proc. SPIE Multimedia Comput. Network., San Jose, CA, Jan. 2002, vol. 4673, pp. 186-195.

[70] T. Nguyen and A. Zakhor, "Distributed video streaming with forward error correction," in 
Proc. Packet Video Workshop, Pittsburgh, PA, Apr. 2002.

[71] J. Chakareski and B. Girod, "Server diversity in rate-distortion optimized streaming of multimedia," in Proc. IEEE ICIP, Barcelona, Spain, Sep. 2003, vol. 3, pp. 645-648.

[72] A. Majumdar, R. Puri, and K. Ramchandran, "Distributed multimedia transmission from multiple servers," in Proc. IEEE ICIP, Rochester, NY, Sep. 2002, vol. 3, pp. 177-180.

[73] J. Kim, R. M. Mersereau, and Y. Altunbasak, "Network-adaptive video streaming using multiple description coding and path diversity," in Proc. IEEE ICME, Baltimore, MD, Jul. 2003, vol. 2, pp. 653-656.

[74] J. Chakareski and P. Frossard, "Distributed collaboration for enhanced sender-driven video streaming," IEEE Trans. Multimedia, to be published.

[75] J. Chakareski and P. Frossard, "Distributed streaming via packet partitioning," in Proc. IEEE ICME, 2006.

[76] J. Chakareski and P. Frossard, "Low-complexity adaptive streaming via optimized a priori media pruning," in Proc. IEEE MMSP, Shanghai, China, Oct./Nov. 2005.

[77] J. C. De Martin and D. Quaglia, "Distortion-based packet marking for MPEG video transmission over diffserv networks," in Proc. IEEE ICME, Tokyo, Japan, Aug. 2001, pp. 521-524.

[78] Y. Sun, I. Sheriff, E. Belding-Royer, and K. Almeroth, "An experimental study of multimedia traffic performance in mesh networks," in Proc. Int. Workshop Wireless Traffic Measure. Model. (WitMeMo), Seattle, WA, Jun. 2005.
[79] C. Chou and A. Misra, "Low latency multimedia broadcast in multi-rate wireless meshes," in Proc. First IEEE Workshop on Wireless Mesh Networks, Sep. 2005.

[80] D. Beyer, N. Waes, and K. Eklund, "Tutorial: 802.16 MAC-layer mesh extensions," in IEEE 802.16 Standard Group Discussions, Feb. 2002.

[81] V. Gunasekaran and F. Harmantzis, "Affordable infrastructure for deploying wimax systems: Mesh v. non mesh," in Proc. IEEE 61st Veh. Technol. Conf. (VTC), May-Jun. 2005, vol. 5, pp. 2979-2983.

[82] R. Bruno, M. Conti, and E. Gregori, "Mesh networks: Commodity multihop ad hoc networks," IEEE Commun. Mag., vol. 43, pp. 123-131, Mar. 2005.

[83] Status of Project IEEE 802.11n, IEEE 802.11-TG N, Jan. 2006. [Online]. Available: http://www.grouper.ieee.org/groups/802/11/ Reports/tgn_update.htm

[84] A. Vasan and A. Shanker, "An empirical characterization of instantaneous throughput in 802.11b WLANs," Univ. of Maryland, Tech. Rep. CS-TR-4389, 2002.

[85] P. Gupta and P. Kumar, "The capacity of wireless networks," IEEE Trans. Inf. Theory, vol. 46, pp. 377-404, Mar. 2000.

[86] J. Li, C. Blake, D. D. Couto, H. Lee, and R. Morris, "Capacity of ad hoc wireless networks," in Proc. 7th Int. Conf. Mobile Comput. Network., Rome, Italy, 2001, pp. 61-69.

[87] I. Akyildiz, X. Wang, and W. Wang, "Wireless mesh networks: A survey," Comput. Netw., vol. 47, pp. 445-487, 2005.

[88] S. Gray, D. Kotz, C. Newport, N. Dubrovsky, A. Fiske, J. Liu, C. Masone, S. McGrath, and Y. Yuan, "Outdoor experimental comparison of four ad hoc routing algorithms," in Proc. 7th ACM Int. Symp. Model., Anal. Simul. Wireless Mobile Syst. (MSWiM), Venice, Italy, Dec. 2004, pp. 220-229.

[89] D. D. Couto, S. Aguayo, B. Chambers, and R. Morris, "Performance of multihop wireless networks: Shortest path is not enough," ACM SIGCOMM Comput. Commun. Rev., vol. 33, pp. 83-88, Jan. 2003.

[90] R. Draves, J. Padhye, and B. Zill, "Comparison of routing metrics for static multi-hop wireless networks," in Proc. ACM Annual Conf. Special Interest Group Data Commun. (SIGCOMM), Aug. 2004, pp. 133-144.

[91] Y. J. Liang, J. G. Apostolopoulos, and B. Girod, "Analysis of packet loss for compressed video: Does burst-length matter?" in Proc. IEEE ICASSP, Apr. 2003, vol. 5, pp. 684-687.

[92] K. Rojviboonchai, F. Yang, Q. Zhang, H. Aida, and W. Zhu, "AMTP: A multipath multimedia streaming protocol for mobile ad hoc networks," in Proc. IEEE ICC, May 2005, vol. 2, pp. 1246-1250.

[93] E. Belding-Royer and A. Lindgren, "Multi-path admission control for mobile ad hoc networks," in Proc. 2nd Annu. Int. Conf. Mobile Ubiquitous Syst. Network. Services (MobiQuitous), Jul. 2005, pp. 407-417.

[94] IEEE Standard for Information Technology Telecommunications and Information Exchange Between Systems-Local and Metropolitan Area Networks-Specific Requirements Part 11: Wireless LAN Medium Access Control (MAC) and Physical Layer (PHY) Specifications Amendment 8: Medium Access Control (MAC) Quality of Service Enhancements, IEEE Std 802.11e, IEEE 802.11 Committee, Nov. 2005.

\section{ABOUT THE AUTHORS}

Pascal Frossard (Senior Member, IEEE) received the M.S. and Ph.D. degrees in electrical engineering from the Swiss Federal Institute of Technology (EPFL), Lausanne, in 1997 and 2000, respectively.

Between 2001 and 2003, he was a Member of Research Staff with the IBM T. J. Watson Research Center, Yorktown Heights, NY, where he worked on media compression and streaming technologies. He is now a tenure-track Assistant Professor

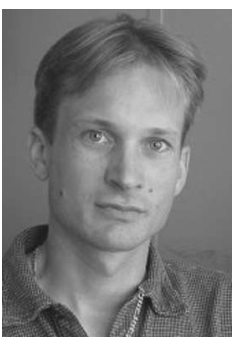
with EPFL, where he heads the LTS4 laboratory in the Signal Processing Institute. His research interests include image representation and coding, nonlinear representations, visual information analysis, joint source and channel coding, multimedia communications, and multimedia content distribution.

Dr. Frossard has been the General Chair of IEEE ICME 2002 (Lausanne) and a member of the organizing or technical program committees of numerous conferences. He was lead Guest Editor of the IEEE JOURNAL ON Selected Areas in Communications Special Issue on "Cross-Layer Optimized Wireless Multimedia Communications" (May 2007) and Guest Editor of the IEEE TRANSACTIONS ON MULTIMEDIA Special Issue on Streaming Media (April 2004). He has been an Associate Editor of the IEEE TRANSACTIONS ON MULTIMEDIa since 2004 and of the IEEE TRANSACTIONS on CIRCUITS AND SYSTEMS FOR VIDEO TECHNOLOGy since 2006. He has been Vice-Chair of the IEEE Multimedia Communications Technical Committee. He serves as a member of the IEEE Image and Multidimensional Signal Processing Technical Committee, the IEEE Multimedia Signal Processing Technical Committee, the IEEE Visual Signal Processing and Communications Technical Committee, and the IEEE Multimedia Systems and Applications Technical Committee. He received the Swiss NSF Professorship Award in 2003 and the IBM Faculty Award in 2005.
Juan Carlos de Martin (Member, IEEE) is an Associate Professor with the Information Engineering School, Politecnico di Torino, Turin, Italy. His research activities are focused on multimedia processing and transmission. He spent two years (1993-1995) as a Visiting Scholar with the Signal Compression Laboratory, University of California-Santa Barbara and two years (19961998) with Texas Instruments, Dallas, as a Member of Technical Staff. He was an Adjunct Professor at

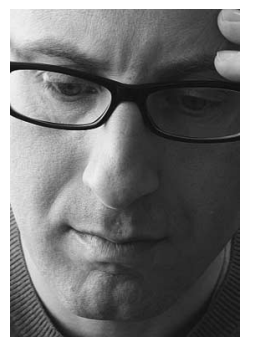
the University of Texas in 1999. Between 1998 and 2005, he was a Principal Researcher with the National Research Council (CNR), Turin, where he led the Multimedia Communications Research Group. He is also active in exploring the interaction between digital technologies and society. In this regard, in November 2006, he founded and currently directs the NEXA Center for Internet and Society of Politecnico di Torino; he is also Coordinator of COMMUNIA, the European thematic network on the digital public domain funded by the European Commission (20072010). He is the author or coauthor of more than 70 international scientific publications; he is also an expert evaluator of research programs for the Italian Ministry of University and Research, for the Ministry of Industrial Activities, and for the Swiss Science Foundation.

Dr. de Martin is a member of the IEEE Multimedia Communications and the IEEE Signal Processing Education Technical Committees. 
M. Reha Civanlar (Fellow, IEEE) received the B.S. and M.S. degrees in electrical engineering from Middle East Technical University, Turkey, and the Ph.D. degree in electrical and computer engineering from North Carolina State University (NCSU), Raleigh, in 1984.

$\mathrm{He}$ is a Vice President and Director of the Media Lab, DoCoMo USA Labs, Palo Alto, CA. He was a Visiting Professor of computer engineering at Koc University, Istanbul, Turkey, from 2002 to 2006. He also led a multinational European research project on threedimensional TV transport and participated in numerous Turkish industrial boards. He serves on the advisory boards of Argela Technologies, Inc., on 3G multimedia systems and Layered Media, Inc., on multipoint videoconferencing. Before these, he was Head of the Visual Communications Research Department, AT\&T Labs-Research, starting in 1991. In the same department, he also held Technology Consultant and Technology Leader positions before heading the group.
Prior to that, he was with Pixel Machines Department, Bell Laboratories, where he worked on parallel architectures and algorithms for image and volume processing and scientific visualization. His career began as a Researcher with the Center for Communications and Signal Processing, NCSU, where he worked on image processing. He has numerous publications, several key contributions to the international multimedia communications standards, and more than 40 patents either granted or pending. His current research interests include packet video systems, networked video and multimedia applications with particular emphasis on the Internet and wireless systems, video coding, 3DTV, and digital data transmissions.

Dr. Civanlar is a member of Sigma Xi. He received a 1985 Senior Award from the IEEE Acoustics, Speech, and Signal Processing Society. He is a Fulbright scholar. He was an Editor of the IEEE TRANSACTIONS ON Communications and the IEEE TRANSActions on Multimedia. Also, he was an Editor of the Journal of Applied Signal Processing and is currently an Editor of Image Communications. He served in the MMSP and MDSP Technical Committees of the IEEE Signal Processing Society. 